\title{
Clearing pigmented insect cuticle to investigate small insects' organs in situ using confocal laser-scanning microscopy (CLSM)
}

\author{
Marco Smolla $^{\mathrm{a}, \mathrm{c}, \mathrm{d}}$, Markus Ruchty ${ }^{\mathrm{b}}$, Manuel Nagel ${ }^{\mathrm{a}, \mathrm{c}}$, Christoph J. Kleineidam ${ }^{\mathrm{a}, *}$ \\ ${ }^{a}$ Department of Biology, Universitätsstraße 10, University of Konstanz, Konstanz 78457, Germany \\ ${ }^{\mathrm{b}}$ Life Science Division, Max Schmidheiny-Strasse 201, Leica Microsystems (Schweiz) AG, Heerbrugg, Switzerland \\ ${ }^{c}$ Department of Behavioral Physiology and Sociobiology, Biozentrum, Am Hubland, University of Würzburg, Würzburg, Germany \\ ${ }^{\mathrm{d}}$ Computational and Evolutionary Biology, Faculty of Life Science, University of Manchester, Manchester, United Kingdom
}

Keywords:

Insect anatomy

Transparent cuticle

Hydrogen-peroxide

Bleaching pigments

Immunocytochemistry

Serotonin

\begin{abstract}
A B S T R A C T
Various microscopic techniques allow investigating structures from submicron to millimeter range, however, this is only possible if the structures of interest are not covered by pigmented cuticle. Here, we present a protocol that combines clearing of pigmented cuticle while preserving both, hard and soft tissues. The resulting transparent cuticle allows confocal laser scanning microscopy (CLSM), which yields high resolution images of e.g. the brain, glands, muscles and fine cuticular structures. Using a fluorescent dye, even single labeled neurons can be visualized and resolved up to an imaging depth of $150 \mu \mathrm{m}$ through the cleared cuticle. Hydrogen peroxide, which was used to clear the cuticle, does not preclude immunocytochemical techniques, shown by successful labeling of serotonin immunoreactive neurons (5HT ir) in the ants' brain. The 'transparent insect protocol' presented here is especially suited for small arthropods where dissection of organs is very demanding and difficult to achieve. Furthermore, the insect organs are preserved in situ thus allowing a more precise three dimensional reconstruction of the structures of interest compared to, e.g., dissected or sectioned tissue.
\end{abstract}

\section{Introduction}

Microscopic techniques allow examining amazingly complex and delicate morphological and neuroanatomical structures even in tiny animals like many insect species. In order to understand the remarkably rich behavioral repertoire of insects and the diversity of adaptations on various levels, morphological and anatomical in vestigations of the neuronal substrate, the motor system and other organs are necessary.

A widely used visualization tool in biology is confocal laser scanning microscopy (CLSM). Compared to conventional light mi croscopy, the restricted depth of focus in CLSM improves resolution and allows optical serial sections of specimens to obtain precise three dimensional information of structures in whole mount preparations. The specimens can only be scanned when they are fully transparent, which is commonly achieved by clearing of fixed soft tissue, using methyl salicylate or benzyl alcohol/benzyl ben zoate (Zucker, 2006). Contemporary examples of the importance of

\footnotetext{
* Corresponding author. Tel.: +497531 884407.

E-mail addresses: Marco.Smolla@postgrad.manchester.ac.uk (M. Smolla), Markus.Ruchty@leica-microsystems.com (M. Ruchty), Manuel.Nagel@unikonstanz.de (M. Nagel), Christoph.Kleineidam@uni-konstanz.de (C.J. Kleineidam).
}

this technique in neurobiology are the standard brain and neuropil atlases of the fruit fly Drosophila melanogaster (Rein et al., 2002; Peng et al., 2011), red flour beetle Tribolium castaneum (Dreyer et al., 2010), hawk moth Manduca sexta (Huetteroth and Schachtner, 2005; El Jundi et al., 2009), and honeybee Apis melli fera (Galizia et al., 1999; Brandt et al., 2005).

Approaches that focus on cuticular structures commonly remove soft tissue using enzymes, potassium hydroxide (KOH), or sodium hydroxide (NaOH) (Galassi et al., 1998; Zill et al., 2000). These studies successfully utilized CLSM to characterize the male genitalia of mosquitos Culex tarsalis (Rein et al., 2002; Klaus et al., 2003), complete legs of cockroaches Periplaneta americana (Zill et al., 2000), as well as the morphology of whole small animals like copepods (Crustacea) (Galassi et al., 1998).

The traditional protocols for CLSM are focused on investigating either soft tissue or cuticle structures in whole mount preparations. So far, they fail to provide specimens where soft tissue can be assessed side by side with cuticle structures. Instead, soft tissue commonly is dissected from the exoskeleton prior to investiga tion, which removes the tissue from its original in situ position and is prone to cause damages to the specimen. Moreover, many ar thropods, like the parasitoid wasp Trichogramma evanescens are so small that dissections of organs are almost impossible to achieve. 
An alternative approach for whole mount preparation is phys ical serial sectioning of specimens in slices of only a few microns, which allows investigation of soft tissues in situ. The general drawback of this approach is the time consuming correction of every single slice for alignment, geometric distortion and staining variation (Streicher et al., 1997). Recent improvements already facilitate the advantage of light microscopy to preserve color in formation of serial sections (Handschuh et al., 2010) and may also overcome the problem of misalignment and geometrical distortion of individual slices due to e.g. Serial Block Face Scanning Electron Microscopy (Denk and Horstmann, 2004) and Automated Tape Collection Ultramicrotomy (Schalek et al., 2011).

In the present study, we describe a clearing protocol that allows the investigation of soft tissue as well as the cuticle, making both, cuticle structures and organs, simultaneously accessible for CLSM investigations. In most arthropods, cuticle structures are strongly pigmented, caused by complex polymerized pigments called melanins. They are integrated into the cuticle during melanization, a stepwise oxidation and reformation of the dopamine based precursors (Andersen et al., 1996; Andersen, 2010, 2011). The pig ments tan cuticle and make it opaque, thus it strongly absorbs light, preventing visual inspections beneath the cuticle. Hydroxyl radicals and $\mathrm{OOH}^{-}$groups were shown to destroy the cuticle pigment melanin (Korytowski and Sarna, 1990). Treating ant cuticle with hydrogen peroxide was reported to remove its pigmentation (Stüben and Linsenmair, 2008). We combined this bleaching treatment with a standard protocol to clear soft tissue.

In our experiments we used heads of various ant species, fixed them and subsequently bleached them, followed by dehydration and clearing. Even bleached cuticle might distort the transmittance of light and may negatively influence the optical resolution of CLSM scans. In order to assess the optical resolution when scanning through bleached cuticle, we stained receptor neurons with a fluorescent dye and scanned their arborization in the brain through the head capsule.

\section{Methods}

\subsection{Animals}

We used adult leaf cutting ants (Atta vollenweideri), collected in 2002 at the Reserva El Bagual, Formosa, Argentina (by M. Bolazzi and O. Geissler), Pheidole fervens ants collected in 2007 in Borneo, Malaysia (by H. Feldhaar), and Formica cunicularia ants collected in 2012 near Konstanz, Germany (by M. Nagel). All animals were reared in an environmental chamber at $25{ }^{\circ} \mathrm{C}$ and $50 \%$ relative humidity in a $12 \mathrm{~h} / 12 \mathrm{~h}$ photoperiod either at the rearing facility of the University of Würzburg (A. vollenweideri, Ph. fervens) or the University of Konstanz (F. cunicularia). A. vollenweideri were fed mainly with privet leaves (Ligustrum vulgaris) and dog rose (Rosa canina). Ph. verfens and F. cunicularia ants were fed with Bhatkar's diet (Bhatkar and Whitcomb, 1970) and cockroaches.

Attini ant species have a pronounced size polymorphism within the worker caste and the fungus tending workers are very small (Hölldobler and Wilson, 1990). For our investigations, we only used Atta workers with a head width $<2 \mathrm{~mm}$ ( $n$ 87). Ph. verfens ants were used for (anterograde) receptor neuron staining, and immu nocytochemical stainings were done with $F$. cunicularia workers.

\subsection{Receptor neuron staining}

In $12 \mathrm{Ph}$. verfens ants, we stained receptor neurons, from the antenna to the antennal lobes (anterograde) with the neuron tracer dextran biotin (D 7135, Molecular Probes, Eugene, USA), in order to investigate whether the bleaching procedure affects the tracer and therefore the ability to use fluorescent dyes. Since this was not the case, we could use the stained neurons to assess whether scanning through bleached cuticle hampers single neuron analysis.

Single ant workers were fixed on a Plexiglas holder with dental wax (surgeon periphery wax, Hereaus Kulzer, Germany), and one antenna was fixed with water based white out correction fluid (Tipp Ex, Bic, France). The antenna was then cross sectioned with a sharp razor blade at the flagellum. About $1 \mu \mathrm{L}$ of dextranbiotin (diluted in water) was applied at the cut end of the antenna using a syringe. The dextranbiotin droplet was applied for $15 \mathrm{~min}$ at the antenna tip and afterward removed with distilled water. Subse quently, the ant was placed in a petri dish with a wet paper tissue and kept for $12 \mathrm{~h}$ at room temperature. After the bleaching step (see below), stained heads were incubated with $1 \mathrm{mg} / \mathrm{ml}$ streptavidin Alexa Fluor 488 (S 11226, Molecular Probes, Eugene, USA) in phosphate buffered saline (PBS, pH 7.2), containing $0.2 \%$ Triton $\mathrm{X}$ 100 for one day at room temperature or 3 days at $4{ }^{\circ} \mathrm{C}$. The vials were then kept in darkness to prevent dye bleaching during dehydration and clearing.

\subsection{Fixation, bleaching and immunocytochemistry}

Ants were carefully decapitated with a sharp razor blade. The heads were transferred immediately to ice cold 4\% para formaldehyde (PFA) in PBS for fixation. The samples were stored for $2 \mathrm{~h}$ at room temperature or one day at $4{ }^{\circ} \mathrm{C}$. After fixation, the specimens were rinsed in PBS ( 3 times 10 min each). Subsequent to the washing step, the heads were transferred into an aqueous so lution of 35\% hydrogen peroxide for 8-10 days, depending on how strongly the cuticle was pigmented. The hydrogen peroxide treat ment turns the cuticle form initially black or brown into a light yellow. When the strongly pigmented ommatidia lose their pigmentation, the heads were washed again in PBS $(3 \times 10 \mathrm{~min})$, and dehydrated in a graded ethanol series $(50 \%, 70 \%, 90 \%, 95 \%$, $3 \times 100 \%$; $10 \mathrm{~min}$ each). Finally, the heads were transferred into methyl salicylate (M 2047, Sigma-Aldrich Chemie GmbH, Stein heim, Germany) for clearing. All solutions were replaced within the same vials containing the specimen, using transfer pipettes.

In order to test whether the hydrogen peroxide treatment prevents further investigations with immunocytochemical tech niques, we treated $8 \mathrm{~F}$. cunicularia heads as described above and we added a 5 hydroxytryptamine (serotonin) antibody staining (5HT ir). The only modification of the protocol was a shorter incubation time with hydrogen peroxide, and we dissected the brain after one day of bleaching. The staining protocol was adapted from Kreissl et al. (2010), and low concentrated hydrogen peroxide $(0.001 \%)$ was used in established protocols for immunocytochemical in vestigations in the past (Kreissl et al., 1994). Following the washing steps, the brains were pre incubated in PBS blocking buffer solution with $0.2 \%$ Triton X 100 and $0.2 \%$ bovine serum albumin (PBS T BSA) for $4 \mathrm{~h}$ at room temperature. The primary rabbit anti serotonin antibody (ICCB56 1, Accurate Chemical \& Scientific Corporation, USA) was applied at 1:500 in PBS T BSA for 4 days at room tem perature, rinsed in PBS ( 5 times $1 \mathrm{~h}$ each) and incubated with DAPI (D 9542, Sigma-Aldrich, US) and the secondary goat anti rabbit antibody conjugated to Alexa 488 (S 11223, Invitrogen, Carlsbad, CA) diluted at 1:1000 and 1:500 in PBS T BSA, respectively.

\subsection{Mounting, CLSM and $3 D$ reconstruction}

For CLSM, the heads were transferred to methyl salicylate filled scanning chambers. These chambers are custom made and built from metal sheets $(0.5-0.8 \mathrm{~mm})$ containing a $1 \mathrm{~cm}$ hole sealed on one side with a glued cover slip. After placing the head in the center 
of the chamber with methyl salicylate, it was sealed with a second cover slip.

The heads were examined at a TCS SP2 AOBS (Leica, Germany) or LSM 510 Meta (Zeiss, Germany) confocal laser scanning microscope (Leica Microsystems AG, Wetzlar, Germany) using a $10 \times$ dry objective (0.40 NA) and a $20 \times$ water immersion objective (0.7 NA and 0.95). The optical sections had a resolution of $1024 \times 1024$ pixel. Optical sections of whole heads were obtained at $\mathrm{z}$ dimensional steps ranging from 1 to $5 \mu \mathrm{m}$. As emission light sources, we used a krypton argon or argon laser (405 $\mathrm{nm}$ and $488 \mathrm{~nm}$, respectively).

Three dimensional surface rendered models were created from optical image stacks using AMIRA 5.2.1 (Mercury Computer Sys tems, Berlin, Germany). The software allows an automated light intensity based segmentation that was used for the cuticle struc tures. Brain neuropiles and glands were manually segmented.

\section{Results}

The absorption, reflection and strong auto fluorescence of the cuticle was reduced to such an extent that organs within the head capsule could be investigated (Fig. 1). In all our bleached prepara tions, the auto fluorescence of cuticle was low enough to analyze soft tissue and cuticle structures side by side (Fig. 1). However, soft tissue just beneath thick cuticle structures, e.g. below the apical head capsule and highly transparent structures close to larger volumes of cuticle, e.g. the mandibular glands, are difficult to detect.

Fixation and dehydration procedures, as used in this study, cause tissue shrinkage, especially during the dehydration prior to the clearing. This was also the case in our preparations. We found shrinkage affecting soft tissue more or less pronounced. Small shrinkage effects can be observed as dark areas e.g. around the calyces of the mushroom bodies (Fig. 1B-D). This kind of soft tissue shrinkage often helps to identify individual structures such as brain neuropiles but, of course, may also change the position of organs due to artificial tensions across tissues.

In some cases, shrinkage caused a much stronger effect and the soft tissue was partially or totally detached from the cuticle. Apparently, the fixed and thus hardened tissue including the in sects' epithelium detached from the endocuticle (not shown).

We tried to reduce shrinkage of soft tissue by using a different fixation agent (zinc formaldehyde, Ott, 2008), and a different dehydration method (graded glycerol series, Ott, 2008). However, all protocols revealed variability in shrinkage artifacts.

Some specimens showed larger areas of the soft tissue that could not be visualized with the CLSM and appeared as dark areas (see Fig. 3A). We think that this is caused by poor dehydration of the tissues, which scatter the light so strongly that it cannot be
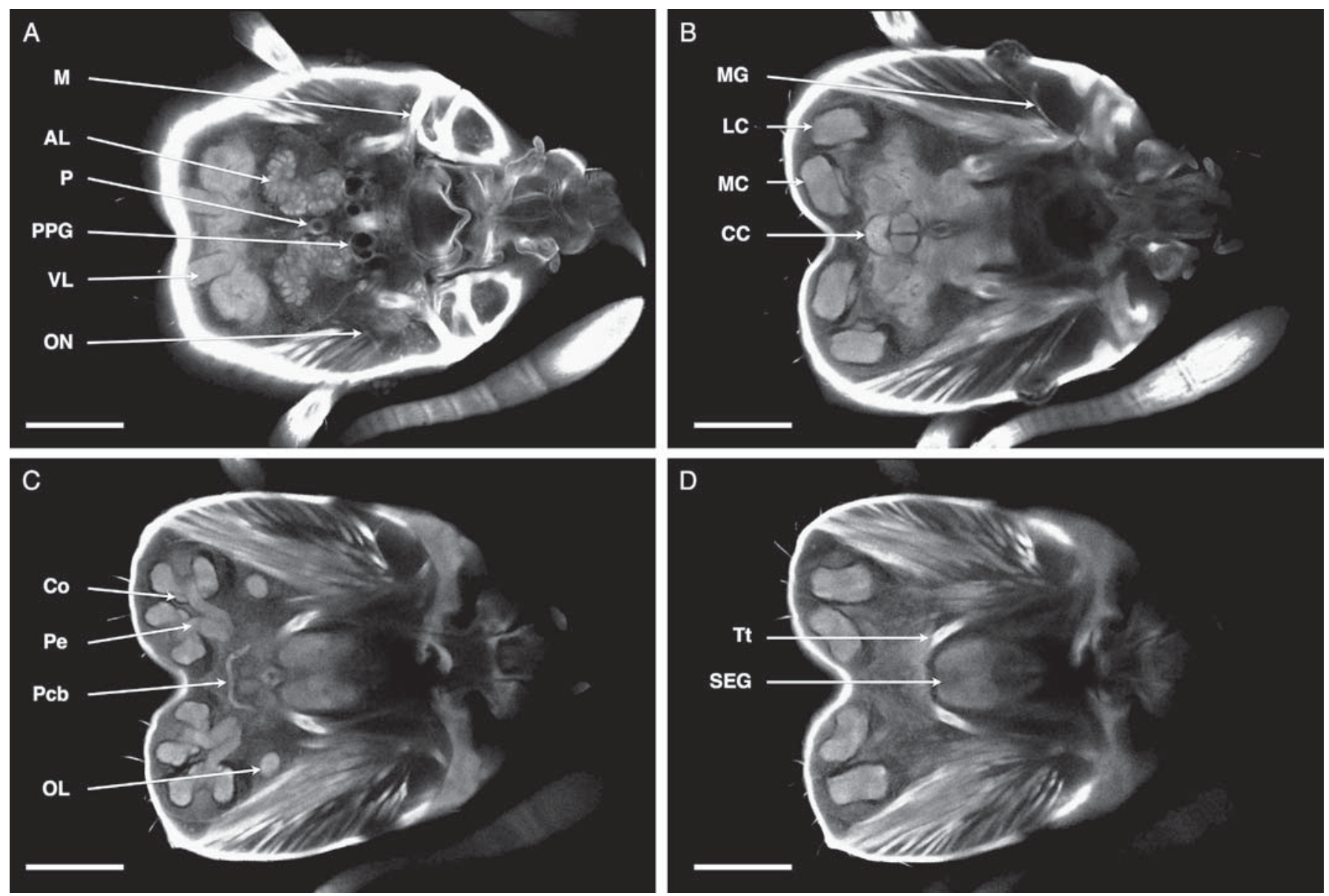

Fig. 1. Confocal images of a head capsule of an Atta vollenweideri worker. Auto-fluorescence images of serial horizontal sections (along the anterior posterior axis) from ventral to dorsal through the head capsule of a single specimen. A: Section at the level of the antennal lobes (AL) and vertical lobes (VL). In this section the optical nerve (ON) of the right eye can be seen as a dim line. Furthermore, the post-pharyngeal gland (PPG), the pharynx (P), and the hollow mandibles (M) can be seen. B: Section at the level of the central complex (CC), the upper part of the lateral and medial calyces (LC, MC) of the mushroom bodies, and the mandibular glands (MG). C: Section at the center of the mushroom body calyces with collar (Co) and peduncle (Pe), optical lobes (OL), and protocerebral bridge (Pcb). D: Section at the lower part of the mushroom bodies, at the level of the subesophageal ganglion (SEG) and the tentorium (Tt). Scale bars: $200 \mu \mathrm{m}$. 

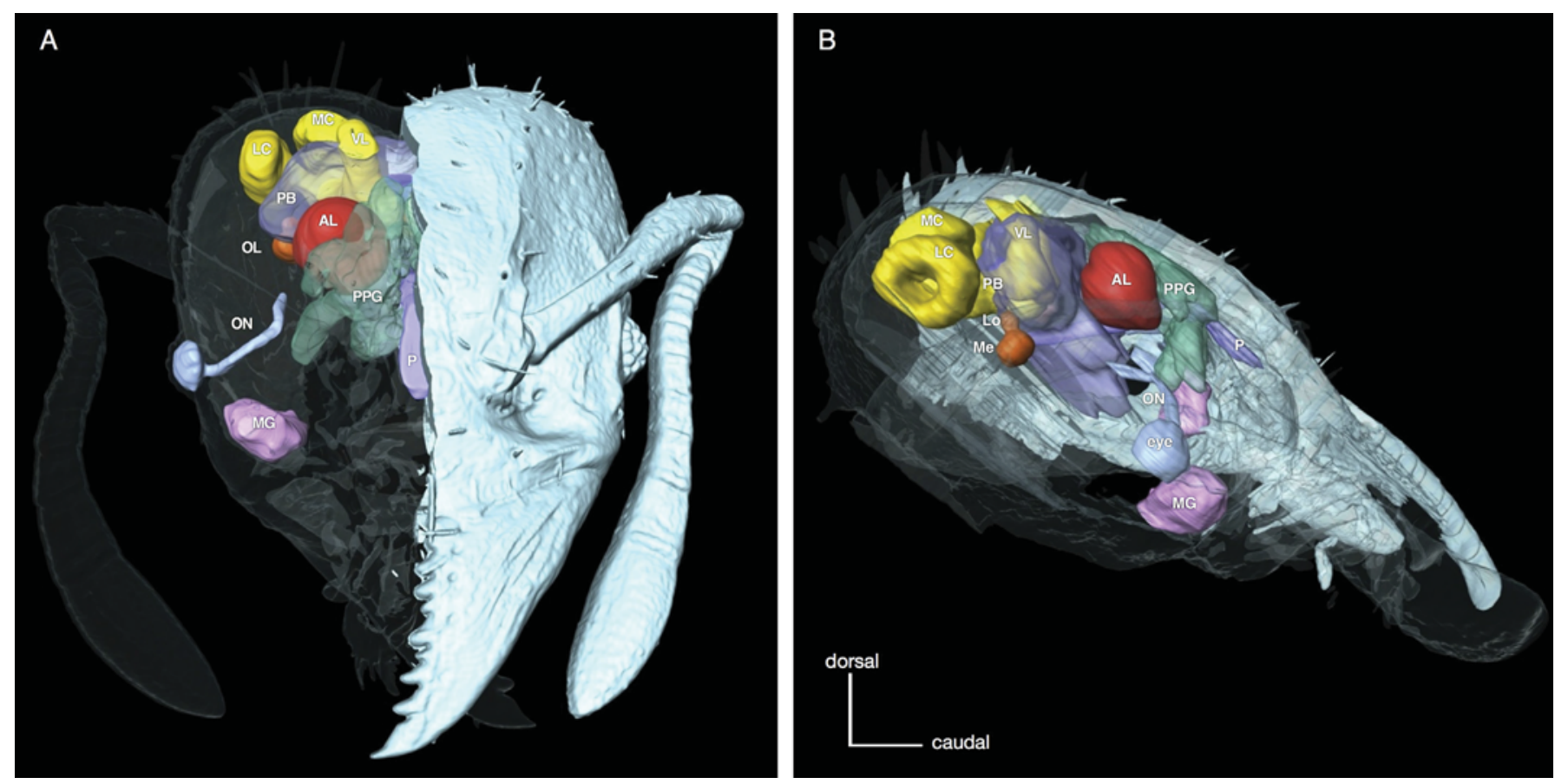

Fig. 2. Surface rendering of an Atta vollenweideri worker head capsule and inner organs. A: frontal view and B: sagital view of the head shown in Fig. 1. Antennal lobe (AL), lateral and medial calyx (LC, MC) of the mushroom body, vertical lobe (VL), protocerebrum (semi transparent, $\mathrm{PB}$ ), optical lobe (OL) with medulla (Me) and lobula (Lo), optical nerve (ON), mandibular and post-pharyngeal gland (MG, PPG), and pharynx (P).

resolved. A poor dehydration might occur if the dehydration agent diffuses insufficiently through the opened occipital foramen at the backside of the head. We tried to circumvent this problem by cut ting small holes in both posterior lateral sides of the heads. How ever, we found that the muscles, attached to this part of the head capsule, were cut as well and pulled the brain out of its natural position, which made us refrain from this method.

Based on our high demands on soft tissue preservation for a high quality brain reconstruction we found 21 out of 83 heads to show sufficiently small shrinkage effects and a good tissue dehydration. We found the auto fluorescence being sufficient to trace individual organs and cuticle structures with the software segmentation ed itor. As an example, we reconstructed the head capsule, brain neuropiles, post pharyngeal gland, both mandibular glands, optical nerves, and eyes of a single head (Fig. 2).

The high auto fluorescence of both inner (tentorium) and outer cuticle structures (antenna joints, ommatidia, hairs) allowed automated cuticle segmentation (Fig. 2A), resulting in a detailed model of the head capsule.

For the reconstruction of soft tissue (Fig. 2), we manually segmented the images based on morphological characteristics (see Fig. 1). We exemplarily reconstructed the post pharyngeal and mandibular gland (Fig. 2A-B). These secretory organs produce and store liquids that scatter light much more than the membrane of the glands. Under CLSM conditions, glands appear as dark struc tures with bright borders (Fig. 1A-B).

About half the volume of the head capsule consists of muscles. The most prominent muscles connect the posterior lateral and the posterior ventral part of the head with the huge mandibles. Mus cles appear as long banded fibrous structures (Fig. 2).

Nervous tissue appeared mainly as homogenous colored struc tures. The most prominent neuropiles in the hymenopteran brain are the mushroom bodies (Fig. 1B-D), antennal lobes (Fig. 1A), central complex (Fig. 1B), and the subesophageal ganglion (Fig. 1CD). In most specimens, all structures could be subdivided into their further compartments (mushroom bodies: calyx, pedunculus, col lar; antennal lobes: glomeruli; central complex: fan shaped and ellipsoid body; protocerebral bridge). We could also identify and reconstruct the protocerebral lobes of the mushroom bodies (the vertical or $\alpha$ lobes, and horizontal or $\beta$ lobes).

In the antennal lobes, auto fluorescence is sufficient to discriminate single glomeruli. However, to better distinguish be tween single glomeruli, we selectively stained antennal lobes by anterograde labeling of receptor neurons at the antenna with a tracer and a fluorescent dye. The axonal projections of the stained neurons project to the dorsal lobe and glomeruli in the antennal lobes. Successfully stained antennal receptor neurons innervated the ipsilateral antennal and dorsal lobe. Due to the staining, the contrast between the antennal lobe and the surrounding tissue was dramatically enhanced (Fig. 3A). Although we scanned through the head capsule, we achieved a resolution that was sufficient to visualize individual neurons within glomeruli up to a depth of $\sim 150 \mu \mathrm{m}$ (Fig. 3C). Hence, the bleaching treatment did neither cause the tracer to leave the receptor neurons nor did it affect the ability of the tracer to bind with the fluorescent dye.

As a proof of principle, we used an antibody staining against serotonin in order to test whether the hydrogen peroxide treat ment interferes with classical immunocytochemical investigations. We found distinct $5 \mathrm{HT}$ ir staining in most parts of the brain, e.g. in the antennal lobe many but not all clusters of glomeruli are innervated (not shown), and in the medial and lateral calyces of the mushroom body the outer lip is innervated (Fig. 4). Some of the 5HT ir neurons are located in a cluster ventral to the medial calyx and some are closer to the midline of the brain, close to the central complex.

\section{Discussion}

The newly developed protocol presented in this study proved suited to visualize inner organs like the brain, glands, and muscles 

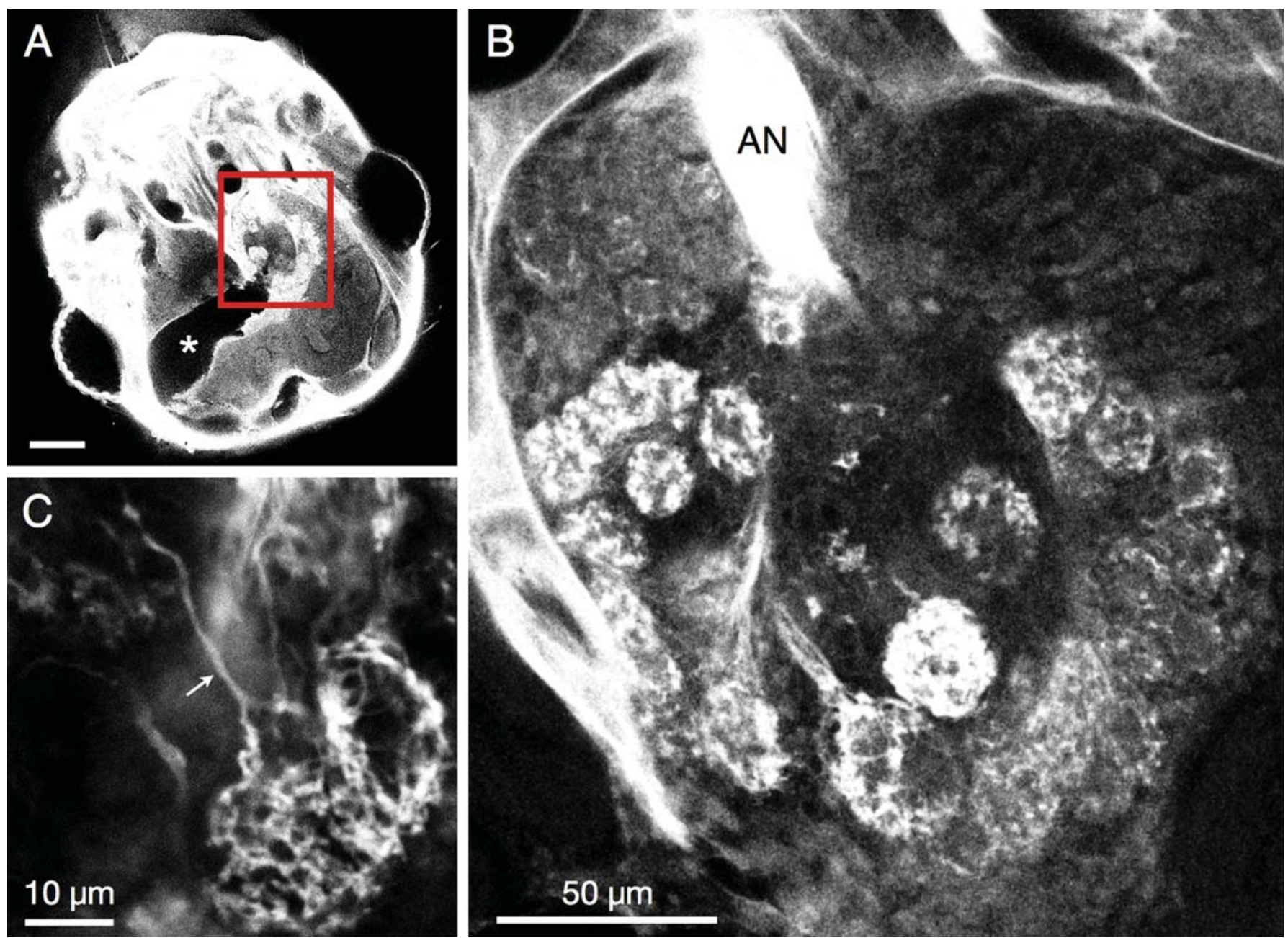

Fig. 3. Optical section of the head of a Pheidole fervens with a fluorescence labeled right antennal lobe. A: Overview of the head with an optical section at the level of the antennal lobes (scale bar: $100 \mu \mathrm{m}$ ). The asterisk indicates tissue that cannot be resolved due to poor dehydration (see text). The red square indicates the area of the image in B. B: The right antennal lobe with a fluorescent staining of receptor neurons. The antennal nerve (AN) enters from the top of the image. The spherical glomeruli can be distinguished very well. C: A higher magnification of the same antennal lobe revealed the arborization of an individual axon (arrow) into a glomerulus at a depth of about $150 \mu \mathrm{m}$.

side by side with cuticle structures. We combined a protocol that was previously used to investigate cuticle structures (Galassi et al., 1998; Michels, 2007; Stüben and Linsenmair, 2008) with traditional protocols for preserving and clearing of soft tissue (Kleineidam et al., 2005). We demonstrate that the used protocol effectively removes tanning pigments and clears soft tissue and cuticle, while it preserves the tissues and even fluorescent dye staining. We were able to visualize single neurons with their arborizations (see Fig. 3C), which demonstrates that scans through the intact head capsule can still gain high resolution images.

We used an excitation wavelength of $488 \mathrm{~nm}$, which yielded the strongest auto fluorescence of the cuticle. This corresponds to related studies on cockroaches (Zill et al., 2000), fruit flies and mosquitos (Klaus et al., 2003; Schawaroch et al., 2005), and co pepods (Michels, 2007). Besides the cuticle, we also find auto fluorescence of soft tissue as has been shown, e.g., for the ejacu latory bulb of Drosophila (Heinstra and Thörig, 1982) and integ umental structures in copepods (Galassi et al., 1998). Due to the fact that the cuticle and soft tissue showed strong auto fluorescence at similar excitation wavelength, we always recorded signals for both at the same time. The cleared cuticle however had a much stronger auto fluorescence compared to unstained soft tissue, which appeared dim, relative to the cuticle. Especially close to the cuticle, soft tissue becomes indistinguishable from the exoskeleton due to its strong over glow effect. The auto fluorescence of the cuticle might be decreased by lower concentration of the fixation agent paraformaldehyde (Zucker, 2006), while the auto fluorescence of soft tissue can be increased by using for example glutaraldehyde. Dissected brains showed a stronger auto fluorescence when they were fixed with a mixture of glutaraldehyde and paraformaldehyde (fix mix, Kelber et al., 2009). How different fixation agents might influence the auto fluorescence of cuticle and soft tissue in the same specimen remains an open question for future studies.

Additionally, our protocol can be combined with standard pro tocols e.g. for anterograde labeling of receptor neurons as well as $5 \mathrm{HT}$ ir neurons. Anterograde labeling of receptor neurons using a fluorescent tracer allows visualization of, e.g., axonal arbors and varicosities at a resolution comparable with results obtained from dissected brains (Kelber et al., 2006; Ruchty et al., 2010). In our immunocytochemical approach, we compared 5HT ir neurons of our specimens ( $F$. cunicularia) with $5 \mathrm{HT}$ ir neurons described in other ant species, and we found similar numbers of neurons at corresponding locations in the protocerebrum (Hoyer et al., 2005; Tsuji et al., 2007). This result indicates that even after the treat ment with 35\% hydrogen peroxide, neurons with immunocyto chemical stainings are maintained. 


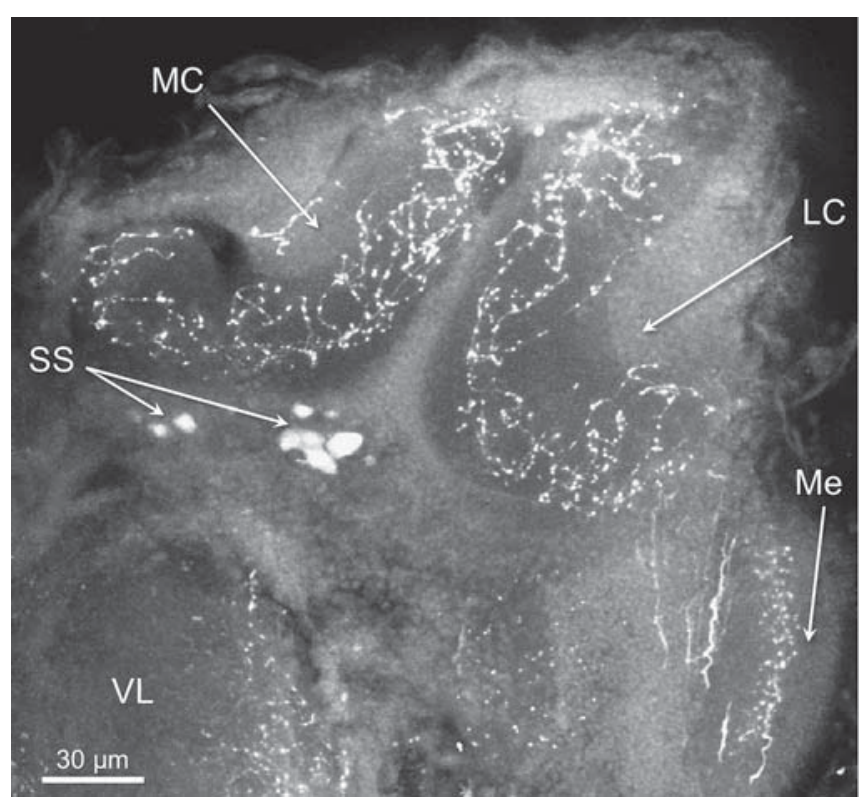

Fig. 4. 5HT-ir neurons in the brain of Formica cunicularia. A projection view of $28 \mu \mathrm{m}$ of the dorsal, left part of the brain is shown. The outer lip of both, the medial and the lateral calyces (MC and $\mathrm{LC}$ ), the medulla (Me), and parts of the vertical lobe (VL) are innervated by 5HT-ir neurons. A cluster of several stained somata (SS) is located ventral to the medial calyx and few are close to the central complex.

All our investigated specimens showed different amounts of shrinkage. Minor shrinkage appeared beneficial for our in vestigations, because organs and soft tissue with low contrast become more separated and condensed, resulting in higher contrast. Stronger shrinkage of soft tissue clearly is undesirable, because it affects the integrity of the in situ observation of inner organs, and makes it necessary to correct size measurements (Bucher et al., 2000).

The majority of the shrinkage is caused by the last dehydration step, when the specimen is transferred into $100 \%$ ethanol (Bucher et al., 2000; Ott, 2008). This transition causes a high osmotic force onto the tissue. Possibly, additional and longer dehydration steps at higher concentration of the ethanol series may reduce the osmotic force and thus the shrinkage.

In additional experiments we countered the impact of soft tissue shrinkage in two ways: using the fixation reagent zinc formaldehyde and dehydrating the specimens with a graded glycerol series. Zinc formaldehyde is reported to preserve tissue in a more rigid form, compared to paraformaldehyde. Glycerol has roughly twice the molar mass of ethanol, and therefore penetrates soft tissue much slower, causing lower osmotic force onto the tissue (Ott, 2008). Unfortunately, we did not notice differences in shrinkage by comparing zinc formaldehyde/glycerol with para formaldehyde/ethanol treated specimens (not shown). However, observing organs, for example the brain, inside the head capsule provides information about what shrinkage actually means. A reliable way to quantify the shrinkage due to fixation and dehy dration protocols requires to assess the initial volume by e.g. using Synchrotron X ray Phase Contrast Microtomography (SR $\mu \mathrm{CT}$, see below), while the final measurement can be achieved with stan dard Confocal Laser Scanning Microscopy (CLSM) as described here.

CLSM is a well established microscopic technique that has become a standard imaging tool in many different disciplines, but especially throughout biology (Amos et al., 1987). It allows scanning through cleared objects, is sensitive to fluorescent dyes, and takes images with submicron resolution.
However, as in all light microscopy, the numerical aperture and the magnification of the used objective constrains the working distance between objective and specimen. We found a penetration depth of up to $500 \mu \mathrm{m}$ acceptable for investigation, which is com parable to other studies (Michels, 2007; Wanninger, 2007). Struc tures of interest that are embedded deeper in tissue suffer from a distinct decrease of fluorescence intensity and appear blurry, due to scattering and absorption of the emitted and reflected light.

A microscopy technique that does not have this constraint is SR $\mu \mathrm{CT}$. It even allows scanning through uncleared specimens pro ducing high resolution images (Betz et al., 2007; Socha and De Carlo, 2008; Montealegre $\mathrm{Z}$ et al., 2012). In contrast to light mi croscopy, SR $\mu \mathrm{CT}$ uses X rays. Thus SR $\mu \mathrm{CT}$ can not detect fluores cent dyes, which are important tools for individually staining cells and tissues (Lichtman and Conchello, 2005).

A relatively recent invention in light microscopy that is able to scan cleared specimens in situ and detect fluorescent dyes is Scan ning Laser Optical Tomography (SLOTy, Lorbeer et al., 2011; Eickhoff et al., 2012). It combines Optical Projection Tomography (OPT, Sharpe, 2002) with a laser. Commonly, a rotating specimen is excited with a laser and scattered, fluorescent, and transmitted light is collected from different viewing angles. Like CLSM, SLOTy works well with cleared specimens. In combination with fluorescent dyes it was applied to study size changes of mushroom bodies and antennal lobes in locust brains (Eickhoff et al., 2012). At the current state of development, the resolution of SLOTy is lower compared to CLSM, and well suited to study specimens up to a couple of milli meters. With an improved optical resolution, SLOTy would have a clear advantage over CLSM because it has not such a strong limita tion of the specimen size. Future studies will aim to combine im aging techniques like SLOTy and the presented protocol to utilize the advantages of both techniques: clearing whole mount pigmented specimens, and scanning the specimen from different angles.

Compared to other methods, the presented protocol has a relatively small workload, and requires neither advanced prepara tion skills nor expensive chemicals. A large number of specimens can be processed at the same time. The relatively long period of bleaching is balanced by the protocol's potential (1) to apply fluo rescent dyes, (2) make in situ observation of inner organs and cuticle structures, and (3) scan images with submicron resolution. The high resolution images are well suited to be analyzed image by image and to create rendered, high resolution three dimensional models that further can be analyzed from different angles and digitally dissected.

We are confident that the presented protocol, in combination with modern microscopy techniques, will become a widely used tool to visualize and analyze arthropod structures in general, and to analyze small arthropods in particular, which might be inac cessible with other methods.

\section{Author contributions}

Conceived and designed the experiments: MR, MS, CJK. Per formed the experiments: MS, MN, MR. Wrote the paper: MS. Pro vided feedback on the manuscript: CJK, MR, MN.

\section{Funding}

This study was supported by the Deutsche For schungsgesellschaft (DFG SFB 554/A6, KL 1327/2).

\section{Acknowledgment}

The authors thank A. Laudahn and A. Gerber Kurz for taking care of several ant colonies. They also thank C. Kelber for helping with 
AMIRA, and identifying and labeling brain neuropiles. They also thank K. Möller for preparation and confocal laser scanning of Pheidole fervens ants.

\section{References}

Amos, W.B., White, J.G., Fordham, M., 1987. Use of confocal imaging in the study of biological structures. Appl. Opt. 26, 32393243.

Andersen, S.O., 2010. Insect cuticular sclerotization: a review. Insect Biochem. Mol. Biol. 40, 166178.

Andersen, S.O., 2011. Cuticular sclerotization and tanning. Insect Biochem. Mol. Biol. 4, 167192.

Andersen, S.O., Peter, M.G., Roepstorff, P., 1996. Cuticular sclerotization in insects. Comp. Biochem. Physiol. Part B: Biochem. Mol. Biol. 113, 689705.

Betz, O., Wegst, U., Weide, D., Heethoff, M., Helfen, L., Lee, W.-K., Cloetens, P., 2007. Imaging applications of synchrotron X-ray phase-contrast microtomography in biological morphology and biomaterials science. I. General aspects of the technique and its advantages in the analysis of millimetre-sized arthropod structure. J. Microsc. 227, 5171.

Bhatkar, A., Whitcomb, W.H., 1970. Artificial diet for rearing various species of ants. Fla. Entomol., 229232.

Brandt, R., Rohlfing, T., Rybak, J., Krofczik, S., Maye, A., Westerhoff, M., Hege, H.-C., Menzel, R., 2005. Three-dimensional average-shape atlas of the honeybee brain and its applications. J. Comp. Neurol. 492, 119.

Bucher, D., Scholz, M., Stetter, M., Obermayer, K., Pflüger, H.J., 2000. Correction methods for three-dimensional reconstructions from confocal images: I. Tissue shrinkage and axial scaling. J. Neurosci. Methods 100, 135143.

Denk, W., Horstmann, H., 2004. Serial block-face scanning electron microscopy to reconstruct three-dimensional tissue nanostructure. PLoS Biol. 2, e329.

Dreyer, D., Vitt, H., Dippel, S., Goetz, B., El Jundi, B., Kollmann, M., Huetteroth, W., Schachtner, J., 2010. 3D standard brain of the red flour beetle Tribolium castaneum: a tool to study metamorphic development and adult plasticity. Front. Syst. Neurosci. $4,3$.

Eickhoff, R., Lorbeer, R.-A., Scheiblich, H., Heisterkamp, A., Meyer, H., Stern, M., Bicker, G., 2012. Scanning laser optical tomography resolves structural plasticity during regeneration in an insect brain. PLoS ONE 7, e41236.

Galassi, D.M.P., De Laurentis, P., Giammatteo, M., 1998. Integumental morphology in copepods: assessment by confocal laser scanning microscopy (CLSM). Fragm. Entomol. (Roma) 30, 7992.

Galizia, C.G., Mcllwrath, S.L., Menzel, R., 1999. A digital three-dimensional atlas of the honeybee antennal lobe based on optical sections acquired by confocal microscopy. Cell. Tissue Res. 295, 383394.

Handschuh, S., Schwaha, T., Metscher, B.D., 2010. Showing their true colors: a practical approach to volume rendering from serial sections. BMC Dev. Biol. 10, 41.

Heinstra, P.W.H., Thörig, G.E.W., 1982. Multiple function of pteridines in Drosophila: the fluorescence of the ejaculatory bulb in Drosophila melanogaster. J. Insect Physiol. 28, 847855

Hölldobler, B., Wilson, E.O., 1990. The Ants, first ed. Belknap Press of Harvard University Press, Cambridge, MA.

Hoyer, S.C., Liebig, J., Rössler, W., 2005. Biogenic amines in the ponerine ant Harpegnathos saltator: serotonin and dopamine immunoreactivity in the brain. Arthropod. Struct. Dev. 34, 429440.

Huetteroth, W., Schachtner, J., 2005. Standard three-dimensional glomeruli of the Manduca sexta antennal lobe: a tool to study both developmental and adult neuronal plasticity. Cell. Tissue Res. 319, 513524.

El Jundi, B., Huetteroth, W., Kurylas, A.E., Schachtner, J., 2009. Anisometric brain dimorphism revisited: implementation of a volumetric 3D standard brain in Manduca sexta. J. Comp. Neurol. 517, 210225.

Kelber, C., Rössler, W., Kleineidam, C.J., 2006. Multiple olfactory receptor neurons and their axonal projections in the antennal lobe of the honeybee Apis mellifera. J. Comp. Neurol. 496, 395405.

Kelber, C., Rössler, W., Kleineidam, C.J., 2009. Phenotypic plasticity in number of glomeruli and sensory innervation of the antennal lobe in leaf-cutting ant workers (A. vollenweideri). Dev. Neurobiol. 70, 222234.

Klaus, A.V., Kulasekera, V.L., Schawaroch, V., 2003. Three-dimensional visualization of insect morphology using confocal laser scanning microscopy. J. Microsc. 212, 107121.
Kleineidam, C.J., Obermayer, M., Halbich, W., Rössler, W., 2005. A macroglomerulus in the antennal lobe of leaf-cutting ant workers and its possible functional significance. Chem. Senses 30, 383392.

Korytowski, W., Sarna, T., 1990. Bleaching of melanin pigments. Role of copper ions and hydrogen peroxide in autooxidation and photooxidation of synthetic dopamelanin. J. Biol. Chem. 265, 1241012416.

Kreissl, S., Eichmüller, S., Bicker, G., Rapus, J., Eckert, M., 1994. Octopamine-like immunoreactivity in the brain and subesophageal ganglion of the honeybee. J. Comp. Neurol. 348, 583595.

Kreissl, S., Strasser, C., Galizia, C.G., 2010. Allatostatin immunoreactivity in the honeybee brain. J. Comp. Neurol. 518, 13911417.

Lichtman, J.W., Conchello, J.-A., 2005. Fluorescence microscopy. Nat. Methods 2, 910919.

Lorbeer, R.A., Heidrich, M., Lorbeer, C., 2011. Highly efficient 3D fluorescence microscopy with a scanning laser optical tomograph. Opt. Express 19, 5419 5430.

Michels, J. 2007. Confocal laser scanning microscopy: using cuticular autofluorescence for high resolution morphological imaging in small crustaceans. J. Microsc. 227, 17.

Montealegre-Z, F., Jonsson, T., Robson-Brown, K.A., Postles, M., Robert, D., 2012. Convergent evolution between insect and mammalian audition. Science 338, 968971.

Ott, S.R., 2008. Confocal microscopy in large insect brains: zinc formaldehyde fixation improves synapsin immunostaining and preservation of morphology in whole-mounts. J. Neurosci. Methods 172, 220230.

Peng, H., Chung, P., Long, F., Qu, L., Jenett, A., Seeds, A.M., Myers, E.W., Simpson, J.H., 2011. BrainAligner: 3D registration atlases of Drosophila brains. Nat. Methods 8 , 493500.

Rein, K., Zöckler, M., Mader, M.T., Grübel, C., Heisenberg, M., 2002. The Drosophila standard brain. Curr. Biol. 12, 227231.

Ruchty, M., Helmchen, F., Wehner, R., Kleineidam, C.J., 2010. Representation of thermal information in the antennal lobe of leaf-cutting ants. Front. Behav. Neurosci. 4, 174.

Schalek, R., Kasthuri, N., Hayworth, K., Berger, D., Tapia, J., Morgan, J., Turaga, S., Fagerholm, E., Seung, H., Lichtman, J., 2011. Development of high-throughput, high-resolution 3D reconstruction of large-volume biological tissue using automated Tape Collection Ultramicrotomy and scanning electron microscopy. Microsc. Microanal. 17, 966967.

Schawaroch, V., Grimaldi, D., Klaus, A.V., 2005. Focusing on morphology: applications and implications of confocal laser scanning microscopy (Diptera: Campichoetidae, Camillidae, Drosophilidae). Proc. Entomol. Soc. Wash. 107, 323 335.

Sharpe, J., 2002. Optical projection tomography as a tool for 3D microscopy and gene expression studies. Science 296, 541545.

Socha, J.J., De Carlo, F., 2008. Use of synchrotron tomography to image naturalistic anatomy in insects. Optical engineering + applications. Int. Soc. Opt. Photonics, 70780A 70780A 7.

Streicher, J., Weninger, W.J., Müller, G.B., 1997. External marker-based automatic congruencing: a new method of 3D reconstruction from serial sections. Anat. Rec. 248, 583602.

Stüben, M., Linsenmair, K.E., 2008. Advances in insect preparation: bleaching, clearing and relaxing ants (Hymenoptera: Formicidae). Myrmecol. News 12, 1521.

Tsuji, E., Aonuma, H., Yokohari, F., Nishikawa, M., 2007. Serotonin-immunoreactive neurons in the antennal sensory system of the brain in the carpenter ant, Camponotus japonicus. Zool. Sci. 24, 836849.

Wanninger, A., 2007. The Application of Confocal Microscopy and 3D Imaging Software in Functional, Evolutionary, and Developmental Zoology: Reconstructing Myo-and Neurogenesis in Space and Time. In: Modern Research and Educational Topics in Microscopy, vol. 1, pp. 353361.

Zill, S., Frazier, S.F., Neff, D., Quimby, L., Carney, M., DiCaprio, R., Thuma, J., Norton, M., 2000. Three-dimensional graphic reconstruction of the insect exoskeleton through confocal imaging of endogenous fluorescence. Microsc. Res. Tech. 48, 367384.

Zucker, R.M., 2006. Whole insect and mammalian embryo imaging with confocal microscopy: morphology and apoptosis. Cytom. Part J. Int. Soc. Anal. Cytol. 69A, 11431152. 